\title{
Dog ownership, dog walking, and leisure- time walking among Taiwanese metropolitan and nonmetropolitan older adults
}

\author{
Yung Liao ${ }^{1 *}$ (D), Pin-Hsuan Huang ${ }^{1}$, Yi-Ling Chen ${ }^{1}$, Ming-Chun Hsueh ${ }^{2}$ and Shao-Hsi Chang ${ }^{2}$
}

\begin{abstract}
Background: This study examined the prevalence of dog ownership and dog walking and its association with leisure-time walking among metropolitan and nonmetropolitan older adults.

Methods: A telephone-based cross-sectional survey targeting Taiwanese older adults was conducted in November 2016. Data related to dog ownership, time spent dog walking (categorized as non-dog owner, non-dog walkers, and dog walkers), and sociodemographic variables were obtained from 1074 older adults. Adjusted binary logistic regression was then performed.

Results: In this sample, $12 \%$ of Taiwanese older adults owned a dog and 31\% of them walked their dogs for an average of 232.13 min over 5.9 days/week (standard deviation = 2.03). Older adults living in nonmetropolitan areas were more likely to own a dog (14.7\% vs. $9.1 \%)$ but less likely to walk their dog (25.9\% vs. $39.6 \%)$ than were those living in metropolitan areas. Compared with non-dog owners, only older adults living in nonmetropolitan areas who were dog walkers achieved 150 min of leisure-time walking (odds ratio: 3.03, 95\% confidence interval: 1.05-8. 77), after adjustment for potential confounders.

Conclusion: Older Taiwanese adults living in nonmetropolitan areas who owned and walked their dogs were more likely to achieve health-enhancing levels of leisure-time walking. Tailored physical activity interventions for promoting dog walking should be developed for older adults who are dog owners living in nonmetropolitan areas and who do not engage in dog walking.
\end{abstract}

Keywords: Walking, Dog ownership, Dog walking, Taiwanese, Older adult

\section{Background}

Physical inactivity is associated with increased risks of mortality and noncommunicable diseases, such as cardiovascular disease, type 2 diabetes, certain types of cancer, and dementia [1, 2]; it also poses a substantial economic burden [3]. In particular, the association between physical inactivity and health outcomes or functional independence is more evident in older adults because older adult populations are the least physically active age group [4]. Although many countries have a national physical activity policy or plan, Sallis et al. [2] reported that physical activity is not increasing worldwide, and that $23.8 \%$ of the global population

\footnotetext{
* Correspondence: liaoyung@ntnu.edu.tw

'Department of Health Promotion and Health Education, National Taiwan Normal University, 162, Heping East Road Section 1, Taipei 106, Taiwan

Full list of author information is available at the end of the article
}

remains physically inactive. In Taiwan, nearly $40 \%$ of Taiwanese older adults failed to achieve the minimum recommendations of physical activity [5]. Therefore, an urgent need exists to develop effective population-based intervention strategies that encourage Taiwanese older adults to engage in sufficient physical activity.

Dog ownership has been widely considered to be a potential method for increasing and maintaining physical activity levels $[6,7]$. A growing body of literature has reported that older adults who are dog owners are more physically active than are non-dog owners or other pet owners $[8,9]$, because dog ownership increases behavioral intention through the dog's positive effect on its owner's cognitive beliefs about walking; moreover, dogs provide an essential form of social support that motivates dog owners to walk [10]. However, previous studies examining associations of dog 
ownership and dog-walking status with physical activity have stated that although dog ownership appears to facilitate walking behavior, only a portion of older dog owners walk their dogs (a range of $22 \%-77 \%$ has been reported by studies in the United Kingdom, the United States, and Japan); nevertheless, those who walked their dogs are more likely to meet the recommended levels of physical activity $[8,11-14]$. Notably, most of these previous studies on older adults have been conducted in well-developed countries, such as the United Kingdom [11], the United States, [8, 12, 13], and Japan [14]; have focused on total physical activity; and have examined samples across the general adult to older adult populations. By contrast, little is known about the prevalence of dog ownership and dog walking and their associations with leisure-time walking among older adults in other cultures and environments, such as Taiwan.

Moreover, the pattern of dog-walking behavior differs between high-density and low-density cities (i.e., dog owners living in low-density cities may be less likely to walk their dog because they have larger yards or public open spaces for dogs to be active without needing to be walked) $[14,15]$. Thus, the prevalence of dog ownership and dog-walking status might differ according to cities with different residential densities (i.e., metropolitan vs. nonmetropolitan areas), and this could be the potential moderator of the associations between dog ownership and walking status and leisure-time walking. However, few studies have examined these associations according to metropolitan and nonmetropolitan areas. Thus, this study first described the prevalence of dog ownership and dog-walking status in metropolitan and nonmetropolitan areas, and then examined the associations of dog ownership and walking status with leisure-time walking among older adults residing in metropolitan and nonmetropolitan areas.

\section{Methods}

\section{Participants}

This study used data from a cross-sectional telephonebased survey, conducted by a research service company using a computer-assisted interviewing system, in November 2016 in Taiwan. It was estimated to have an older adult population of $6,142,472$ with an area of 36,192 . $8 \mathrm{~km}^{2}$ in Taiwan. The needed sample size for the present study was 1067 older adults, which was calculated using a 95\% confidence level and a 3\% confidence interval. A standardized questionnaire was administered by trained interviewers, who had experience in administering telephone population surveys and received 2 days of training before the start of each survey. The response rate was $30.3 \%$ (3546 older adults were contacted, and 1074 of them finished the survey). The telephone research service company did not offer any rewards for participation. Verbal informed consent was received before the beginning of each telephone interview. The protocols of this study were reviewed and approved by the Research Ethics Committee of National Taiwan Normal University (REC number: 201605HM006).

\section{Dog ownership and dog-walking status}

Dog-related variables in the present study were dog ownership and dog-walking behavior. Participants were asked if they owned a dog (yes/no), and if so, whether they walked the $\operatorname{dog}$ (yes/no), the usual frequency of walks (days/weeks), and the duration of the walks (minutes/day). These validated items measuring dog-walking frequency and duration for older adults have been used in previous studies [13, 14]. Dog ownership and walking status were categorized (using dog ownership and time spent dog walking) into three groups: (a) non-dog owners, (b) nondog walkers (defined as dog owners who did not walk their dog), and (c) dog walkers (defined as dog owners who walked their dogs for at least $10 \mathrm{~min} /$ week) $[13,14]$.

\section{Leisure-time walking}

Time spent in leisure-time walking was obtained using the Taiwanese version of the International Physical Activity Questionnaire-long version (IPAQ-LV) [16], which is widely used for telephone-based surveys among middleto-older adults $[17,18]$. IPAQ-LV had a high test-retest reliability $(r=0.78)$ and acceptable criterion validity $(r=0$. 31-0.41) in comparison with accelerometers [16]. Time spent in leisure-time walking was measured using the fourth part of the IPAQ-LV, and was calculated by multiplying the frequency (per week) by the duration of leisure time (per day). Because the distributions were skewed, the outcome variable was categorized on the basis of the IPAQ scoring protocol [19]. Furthermore, based on the physical activity recommendation for older adults [20], the outcome variable was dichotomized into "achieving 150 $\mathrm{min} /$ week" and "not achieving $150 \mathrm{~min} /$ week."

\section{Sociodemographic variables}

The sociodemographic variables included gender, age, type of occupation, educational level, marital status, living status, residential area, self-rated health status, and body mass index (BMI). Age was categorized into three categories: 65 to 74 years, 75 to 84 years, and older than 85 years. Occupational type was classified into "full-time job" and "not full-time job." Educational level was divided into: "non-tertiary degree" ( $<13$ years) and "tertiary degree" ( $\geq 13$ years). Marital status was categorized as "married" and "unmarried" (including widowed, separated, and divorced). Living status was divided into "living with others" and "living alone," and residential area was categorized into "metropolitan" and "nonmetropolitan" areas. Self-rated health status was categorized into "good" and "poor," and BMI score was based on self-reported weight and height and grouped into two 
categories: "not overweight" $\left(<24 \mathrm{~kg} / \mathrm{m}^{2}\right)$ and "overweight/ obese" $\left(\geq 24 \mathrm{~kg} / \mathrm{m}^{2}\right)$ according to Taiwanese cut-off points.

\section{Statistical analysis}

The data of 1074 older adults who had complete information for the study variables were analyzed. Chi-squared tests were used to evaluate the proportional differences in sociodemographic variables and Mann-Whitney U tests were used for continuous variables between the residents of metropolitan and nonmetropolitan areas. In addition, forced-entry adjusted logistic regression was conducted to estimate the odds ratios (ORs) of achieving $150 \mathrm{~min}$ of leisure-time walking according to the categories of dog ownership and dog-walking status, after adjustment for gender and age (Model 1) and the other potential confounders (Model 2); notably, this analysis also examined all of the respondents as one group, and the respondents stratified by metropolitan or nonmetropolitan area. Adjusted ORs and 95\% CIs were calculated for each variable. Inferential statistics were obtained using IBM SPSS 23.0, and the level of significance was set at $p<0.05$.

\section{Results}

Sociodemographic characteristics of the participants

Table 1 contains the sociodemographic characteristics in the total sample according to metropolitan and nonmetropolitan areas. Overall, the mean (standard deviation [SD]) age of the respondents was $72.51( \pm 6.2)$ years. $50.3 \%$ of respondents were men, $65.2 \%$ were aged $65-74$ years, $28.5 \%$ had a tertiary degree, $10.1 \%$ had a full-time job, $76.8 \%$ were married, $86.4 \%$ were living with others, $20.0 \%$ of respondents reported health status were poor, and $41.8 \%$ were either overweight/obese. A total of $49.0 \%$ respondents lived in metropolitan areas. Chi-square test analysis revealed that older adults living in nonmetropolitan area were more likely to be male $(54.7 \%$ vs.45.6\%), not having tertiary education (75.0\% vs.67.9\%), living alone (16.2\% vs.10.8\%) and reporting poor self-rated health status (23.9\% vs.16.0\%).

\section{Prevalence of dog ownership and dog walking status}

Table 2 indicates that of the respondents, $88.0 \%$ were non-dog owner, and $12.0 \%$ owned a dog (including $8.3 \%$ of non-dog walker and $3.7 \%$ of dog walkers). The prevalence of achieving $150 \mathrm{~min} /$ week of leisure-time walking was 53.1\%; on average, the respondents spent 218 . $38 \mathrm{~min}$ in leisure-time walking over 5.98 days/week (SD $=1.80$ ). Additionally, the prevalence of dog ownership was $12 \%$, and $31 \%$ of those respondents walked their dogs for an average of $232.13 \mathrm{~min}$ over 5.9 days/week $(\mathrm{SD}=2.03)$. Chi-squared tests also revealed that older adults living in nonmetropolitan areas were more likely to be a non-dog walker (those who owned a dog but did not walk their dog) than those living in metropolitan areas $(10.9 \%$ vs. $5.5 \%)$. No significant associations were identified for achieving 150 min of leisure-time walking between residents of metropolitan and nonmetropolitan areas. Mann-Whitney $U$ test showed that older adults living in metropolitan areas were more likely to engage in leisure-time walking than were those living in nonmetropolitan areas.

\section{Dog ownership and dog-walking status associated with $150 \mathrm{~min}$ of leisure-time walking in metropolitan and nonmetropolitan areas}

The ORs for attaining $150 \mathrm{~min} /$ week leisure-time walking are presented in Table 3, according to the categories of dog ownership and dog-walking status among the residents of metropolitan and nonmetropolitan areas. Moreover, Table 3 shows that the respondents who were dog walkers living in nonmetropolitan areas were more likely to achieve $\geq 150 \mathrm{~min} /$ week of leisure-time walking according to both Model $1(\mathrm{OR}=3.21 ; 95 \% \mathrm{CI}=1.16-8.95)$ and Model 2 $(\mathrm{OR}=3.03 ; 95 \% \mathrm{CI}=1.05-8.77)$.

\section{Discussion}

This is the first study to provide evidence for the prevalence of dog ownership and dog walking among Taiwanese older adults, stratified by metropolitan or nonmetropoli$\tan$ area. Among the respondents, the prevalence of dog ownership was $12 \%$, and $28.8 \%$ of them walked their dogs for an average of $232.13 \mathrm{~min}$ over 5.9 days/week. The prevalence of dog ownership among Taiwanese older adults (12\%) is slightly lower than that reported in Japan (14\%) [14], the United States (15\%-16\%) [8, 12, 13], and the United Kingdom (22\%) [11]. Moreover, the prevalence of dog walking in our sample was $31.0 \%$, which is considerably lower than that reported in the United States (48\%) and Japan (71\%).

Stratification by area indicated that older adults living in nonmetropolitan areas were more likely to own a dog but less likely to walk their dog than were those living in metropolitan areas. Higher dog ownership in nonmetropolitan areas could be because older adults living in nonmetropolitan areas are more likely to live alone [21]; therefore, they are more likely to own a dog for companionship or security. Moreover, the lower prevalence of dog walking among older adults in nonmetropolitan areas could be because nonmetropolitan areas are characterized by low-density neighborhoods, and older adults are likely to live in detached single-family housing with larger yards; this provides a place for dogs to be active without needing to be walked $[15,22]$.

Another novel finding of the present study highlights how metropolitan status may be a modifier between the categories of dog ownership and dogwalking status and leisure-time walking among older adults. Previous results for well-developed countries have indicated that dog walkers were more likely to 
Table 1 Characteristics of the respondents, according to metropolitan and nonmetropolitan areas

\begin{tabular}{|c|c|c|c|c|}
\hline \multirow[t]{2}{*}{ Characteristics } & \multirow{2}{*}{$\begin{array}{l}\text { Total } \\
\text { N (\%) } 1074(100 \%)\end{array}$} & \multirow{2}{*}{$\begin{array}{l}\text { Metropolitan area } \\
\text { N (\%) } 526(49.0 \%)\end{array}$} & \multirow{2}{*}{$\begin{array}{l}\text { Non-Metropolitan area } \\
\text { N (\%) } 548(51.0 \%)\end{array}$} & \multirow[t]{2}{*}{$P$-value } \\
\hline & & & & \\
\hline Age, mean (SD) & $72.51(6.2)$ & $72.26(6.23)$ & $72.74(6.25)$ & \\
\hline Population density & 650.16 & 1490.04 & 288.06 & \\
\hline Gender & & & & $0.003^{*}$ \\
\hline Male & $50.3 \%$ & $45.6 \%$ & $54.7 \%$ & \\
\hline Female & $49.7 \%$ & $54.4 \%$ & $45.3 \%$ & \\
\hline Age & & & & .578 \\
\hline $65-74$ & $65.2 \%$ & $66.7 \%$ & $63.7 \%$ & \\
\hline $75-84$ & $28.7 \%$ & $27.4 \%$ & $29.9 \%$ & \\
\hline $85+$ & $6.1 \%$ & $5.9 \%$ & $6.4 \%$ & \\
\hline Occupational type & & & & .276 \\
\hline Full-time job & $10.1 \%$ & $9.1 \%$ & $11.1 \%$ & \\
\hline Not full-time job & $89.9 \%$ & $90.9 \%$ & $88.9 \%$ & \\
\hline Education & & & & $0.010^{*}$ \\
\hline Not Tertiary degree & $71.5 \%$ & $67.9 \%$ & $75.0 \%$ & \\
\hline Tertiary degree & $28.5 \%$ & $32.1 \%$ & $25.0 \%$ & \\
\hline Marital status & & & & .250 \\
\hline Unmarried & $23.2 \%$ & $21.7 \%$ & $24.6 \%$ & \\
\hline Married & $76.8 \%$ & $78.3 \%$ & $75.4 \%$ & \\
\hline Living status & & & & $0.010^{*}$ \\
\hline Alone & $13.6 \%$ & $10.8 \%$ & $16.2 \%$ & \\
\hline With others & $86.4 \%$ & $89.2 \%$ & $83.8 \%$ & \\
\hline Self-rated health status & & & & $0.001^{* *}$ \\
\hline Good & $80.0 \%$ & $84.0 \%$ & $76.1 \%$ & \\
\hline Poor & $20.0 \%$ & $16.0 \%$ & $23.9 \%$ & \\
\hline BMI (kg/m2) & & & & .328 \\
\hline Non-overweight & $58.2 \%$ & $59.7 \%$ & $56.8 \%$ & \\
\hline Overweight/Obese & $41.8 \%$ & $40.3 \%$ & $43.2 \%$ & \\
\hline
\end{tabular}

$S D$ standard deviation; $\mathrm{min} / \mathrm{d}$, minutes/day

The proportional difference in characteristics between the residents of metropolitan and nonmetropolitan areas was tested using $\mathrm{X} 2$

${ }^{*} p<0.05,{ }^{* *} p<0.001$

meet the recommended physical activity guideline $[8$, 11-14]; however, our results suggest that dog-walking only appears to be more critical for nonmetropolitan older adults to engage in health-enhancing levels of recreational walking, compared with metropolitan older adults. A possible explanation for these results is that, compared with older adults living in metropolitan areas, those living in nonmetropolitan areas may have lower walkability because of poorer infrastructure (i.e., sidewalks, intersections) and less leisure options (i.e., parks, leisure facilities) in their neighborhood environment to engage in leisure-time walking [23]. Limited leisure options in the neighborhood, may facilitate the accumulation of sufficient leisuretime walking among nonmetropolitan older adults who walk their dogs regularly.
Critically, the aforementioned results indicate that promoting the initiation of dog walking among older adults who live in nonmetropolitan areas and own dogs, but who do not currently walk their dogs, could be a favorable population-based intervention strategy to achieve the recommended levels of leisure-time walking. It is also noticed that older adults living in nonmetropolitan areas were more likely to have the characteristics of living alone and reporting poor self-rated health status. Thus, effective dog-walking intervention strategies, such as changing the perceptions regarding exercise requirements of the dog or providing dog-supportive physical environments, have been suggested as modifiable points for intervention [24, 25]. These dog-walking intervention strategies targeting nonmetropolitan older adults should be considered for future physical activity intervention studies. 
Table 2 Dog ownership, dog-walking status, and leisure-time walking, according to metropolitan and nonmetropolitan areas

\begin{tabular}{|c|c|c|c|c|}
\hline \multirow[t]{2}{*}{ Variable } & Total & Metropolitan area & Non-Metropolitan area & \multirow[t]{2}{*}{$P$-value } \\
\hline & 1074 & $526(49.0 \%)$ & $548(51.0 \%)$ & \\
\hline$\%$ of dog ownership /dog walking category & & & & $.004^{*}$ \\
\hline Non-dog owner & $88.0 \%$ & $90.9 \%$ & $85.2 \%$ & \\
\hline Non-dog walker & $8.3 \%$ & $5.5 \%$ & $10.9 \%$ & \\
\hline Dog walker & $3.7 \%$ & $3.6 \%$ & $3.8 \%$ & \\
\hline$\%$ of dog walking in dog owners & & & & .105 \\
\hline \multicolumn{5}{|l|}{ Dog owner } \\
\hline - Non-dog walker & $69.0 \%$ & $60.4 \%$ & $74.1 \%$ & \\
\hline - Dog walker & $31.0 \%$ & $39.6 \%$ & $25.9 \%$ & \\
\hline$\%$ of meeting 150 min of LT walking & & & & .053 \\
\hline$<150 \min$ & $46.9 \%$ & $43.9 \%$ & $49.8 \%$ & \\
\hline $150+\min$ & $53.1 \%$ & $56.1 \%$ & $50.2 \%$ & \\
\hline Time spent in dog walking (dog walker) & & & & 0.15 \\
\hline Day/Week & 5.85 & 5.21 & 6.43 & \\
\hline Mean & 232.13 & 182.63 & 276.90 & \\
\hline (SD) (min/week) & $(211.11)$ & $(144.57)$ & $(252.31)$ & \\
\hline Time spent in leisure-time walking & & & & $0.03^{*}$ \\
\hline Day/Week & 5.98 & 5.85 & 6.11 & \\
\hline Mean & 218.38 & 232.14 & 205.16 & \\
\hline (SD) (min/week) & (249.34) & (248.86) & (249.32) & \\
\hline
\end{tabular}

$S D$ standard deviation, $L T$ leisure time

Data are expressed as number (percentage) or mean (SD)

Difference across metropolitan area categories was tested using $x^{2}$ for categorical variables and Mann-Whitney $U$ test for continuous variables " $p<0.05$

Table 3 Dog ownership and dog-walking status associated with 150 min of leisure-time walking among all respondents

\begin{tabular}{|c|c|c|}
\hline \multirow[t]{2}{*}{ Variable } & \multicolumn{2}{|c|}{ Leisure-time walking (meeting $150 \mathrm{~min} /$ week) } \\
\hline & $\begin{array}{l}\text { Model } 1 \\
\text { OR (95\% Cl) }\end{array}$ & $\begin{array}{l}\text { Model } 2 \\
\text { OR }(95 \% \text { Cl) }\end{array}$ \\
\hline \multicolumn{3}{|l|}{ Total } \\
\hline Non dog owners & 1.00 (Ref.) & 1.00 (Ref.) \\
\hline Non dog walkers & $0.90(0.58-1.39)$ & $1.05(0.67-1.65)$ \\
\hline Dog walkers & $1.86(0.95-3.66)$ & $1.84(0.92-3.65)$ \\
\hline \multicolumn{3}{|c|}{ Non-Metropolitan area } \\
\hline Non dog owners & 1.00 (Ref.) & 1.00 (Ref.) \\
\hline Non dog walkers & $1.03(0.60-1.77)$ & $1.10(0.63-1.93)$ \\
\hline Dog walkers & $3.21(1.16-8.95)^{*}$ & $3.03(1.05-8.77)^{*}$ \\
\hline \multicolumn{3}{|l|}{ Metropolitan area } \\
\hline Non dog owners & 1.00 (Ref.) & 1.00 (Ref.) \\
\hline Non dog walkers & $0.79(0.37-1.68)$ & $0.91(0.41-1.99)$ \\
\hline Dog walkers & $1.10(0.43-2.80)$ & $1.12(0.43-2.91)$ \\
\hline
\end{tabular}

$O R$ odds ratio, $\mathrm{Cl}$ confidence interval Model 1 is adjusted for gender and age

Model 2 is adjusted for gender, age, city of residence, education level, type of occupation, marital status, living status, BMl, and self-rated health status " $p<0.05$
Several limitations should be addressed for the present study. First of all, the limited sample size for subgroup analysis may decrease the statistical power of our findings. However, the small sample size of subgroup analysis may reflect current situation of dog walking among Taiwanese older adults. Thus, future studies using prospective design with larger sample size should further investigate these associations in this context. Second, because of the cross-sectional design, we could not draw conclusions regarding the causal relationship between dog walking and the recommended levels of leisure-time walking. Third, the measurements of the present study were self-reported and thus subject to bias [16, 26]. Fourth, information of dog owners' preference for dog breeds and dog characteristics such as dog health, age, type, or size that may be related to dog owners' walking behavior [27], were not obtained in this study. Finally, due to the telephone-based survey, this study had a limited representative sample; therefore, sections of the population without a household telephone (approximately $7.1 \%$ in 2015) were impossible to reach [28]. Thus, the present findings may not be generalizable to the overall older adult population in Taiwan. 


\section{Conclusion}

Older Taiwanese adults living in nonmetropolitan areas who owned and walked their dogs were more likely to achieve health-enhancing levels of leisure-time walking. Thus, tailored physical activity interventions for promoting dog walking should be developed for older adults who are dog owners living in nonmetropolitan areas and who do not engage in dog walking. Future studies using qualitative design are needed as an extension from this study to find out the reasons why dog owners not walking their dogs both in metropolitan and non-metropolitan area.

\section{Abbreviations}

BMl: Body mass index; Cl: Confidence interval; IPAQ-LV: International Physical Activity Questionnaire-long version; LT: Leisure time; OR: Odds ratio;

SD: Standard deviation

\section{Acknowledgements}

Not applicable

\section{Funding}

YL had a personal grant from the Ministry of Science and Technology of Taiwan (MOST 105-2410-H-003-050). The Ministry of Science and Technology of Taiwan was not involved in the study design, data collection, analysis, interpretation and writing the manuscript.

\section{Availability of data and materials}

The dataset used and analyzed during the current study are available from the corresponding author on reasonable request

\section{Authors' contributions}

All authors contributed equally to this work. YL conceived and designed the survey, determined the analytical methods and wrote the main paper. YLC and $\mathrm{PHH}$ performed the statistical analyses and participated in the interpretation of the results. MCH helped to collect data and the manuscript preparation. SHC coordinated the survey and critically reviewed the final version of the manuscript. All authors discussed the results and implications and commented on the manuscript at all stages. All authors read and approved the final manuscript.

\section{Ethics approval}

The protocols of this study were reviewed and approved by the Research Ethics Committee of National Taiwan Normal University (REC number: 201605HM006). Because of no sensitive questions or personal information, this telephone-based survey has been evaluated as minimal risk that qualify for a verbal consent process by Research Ethics Committee of National Taiwan Normal University. The process of verbal consent was documented including logging the date, time, and name of a caller, a note that the prescribed script was used, and a record of the participant's response.

\section{Consent for publication}

Not applicable

\section{Competing interests}

The authors declare that they have no competing interests.

\section{Publisher's Note}

Springer Nature remains neutral with regard to jurisdictional claims in published maps and institutional affiliations.

\section{Author details}

'Department of Health Promotion and Health Education, National Taiwan Normal University, 162, Heping East Road Section 1, Taipei 106, Taiwan. ${ }^{2}$ Department of Physical Education, National Taiwan Normal University, Taipei 106, Taiwan
Received: 20 October 2017 Accepted: 21 March 2018

Published online: 04 April 2018

\section{References}

1. Lee IM, Shiroma EJ, Lobelo F, Puska P, Blair SN, Katzmarzyk PT. Lancet physical activity series working group. Effect of physical inactivity on major non-communicable diseases worldwide: an analysis of burden of disease and life expectancy. Lancet. 2012;380:219-29.

2. Sallis JF, Bull F, Guthold R, et al. Progress in physical activity over the Olympic quadrennium. Lancet. 2016; (published online July 27.) https://doi. org/10.1016/S0140-6736(16)30581-5

3. Ding D, Lawson KD, Kolbe-Alexander $T L$, et al. The economic burden of physical inactivity: a global analysis of major non-communicable diseases. Lancet. 2016; (published online July 27.) https://doi.org/10.1016/S01406736(16)30383-X

4. World Health Organization (WHO). Global recommendations on physical activity for health. Geneva: World Health Organization; 2010.

5. Hsueh MC, Liao Y, Chang SH. Perceived neighborhood and home environmental factors associated with television viewing among Taiwanese older adults. Int J Environ Res Public Health. 2016;13(7).

6. Christian HE, Westgarth C, Bauman A, Richards EA, Rhodes RE, Evenson KR, Mayer JA, Thorpe RJ Jr. Dog ownership and physical activity: a review of the evidence. J Phys Act Health. 2013;10(5):750-9.

7. Cutt H, Giles-Corti B, Knuiman M, Burke V. Dog ownership, health and physical activity: a critical review of the literature. Health Place. 2007;13(1):261-72.

8. Thorpe RJ Jr, Kreisle RA, Glickman LT, Simonsick EM, Newman AB, Kritchevsky S. Physical activity and pet ownership in year 3 of the health ABC study. J Aging Phys Act. 2006a;14(2):154-68.

9. Enmarker I, Hellzén O, Ekker K, Berg AG. Health in older cat and dog owners: the Nord-Trondelag health study (HUNT)-3 study. Scand J Public Health. 2012:40(8):718-24. https://doi.org/10.1177/1403494812465031.

10. Cutt HE, Knuiman MW, Giles-Corti B. Does getting a dog increase recreational walking? Int J Behav Nutr Phys Act. 2008;5:17.

11. Harries TJ, Owen CG, Victor CR, Adams R, Cook DG. What factors are associated with physical activity in older people, assessed objectively by accelerometry? Br J Sports Med. 2009;43(6):442-50.

12. Gretebeck KA, Radius K, Black DR, Gretebeck RJ, Ziemba R, Glickman LT. Dog ownership, functional ability, and walking in community-dwelling older adults. J Phys Act Health. 2013;10(5):646-55.

13. Thorpe RJ Jr, Simonsick EM, Brach JS, et al. Dog ownership, walking behavior, and maintained mobility in late life. J Am Geriatr Soc. 2006b;54(9):1419-24.

14. Shibata A, Oka K, Inoue S, Christian H, Kitabatake Y, Shimomitsu T. Physical activity of Japanese older adults who own and walk dogs. Am J Prev Med. 2012:43(4):429-33.

15. Coleman KJ, Rosenberg DE, Conway TL, et al. Physical activity, weight status, and neighborhood characteristics of dog walkers. Prev Med. 2008;47(3):309-12.

16. Liou YM, Jwo CJ, Yao KG, Chiang LC, Huang LH. Selection of appropriate Chinese terms to represent intensity and types of physical activity terms for use in the Taiwan version of IPAQ. J Nurs Res. 2008;16(4):252-63.

17. Hsueh MC, Liao Y, Chang SH. Associations of Total and domain-specific sedentary time with type 2 diabetes in Taiwanese older adults. J Epidemiol. 2016;26(7):348-54

18. Liao Y, Wang IT, Hsu HH, Chang SH. Perceived environmental and personal factors associated with walking and cycling for transportation in Taiwanese adults. Int J Environ Res Public Health. 2015;12(2):2105-19.

19. IPAQ Scoring protocol. International Physical Activity Questionnaire Web site. https://sites.google.com/site/theipaq/scoring-protocol (Accessed 15 Sept 2015).

20. Nelson ME, Rejeski WJ, Blair SN, Duncan PW, Judge JO, King AC, et al. Physical activity and public health in older adults: recommendation from the American College of Sports Medicine and the American Heart Association. Med Sci Sports Exerc. 2007;39(8):1435-45.

21. Ministry of Health and Welfare (2013). Report of the Senior Citizen Condition Survey 2013. Website: file:///C:/Users/victor/Downloads/5. \%E8\%AA\%BF\%E6\%9F\%A5\%E5\%88\%86\%E6\%9E\%90.pdf (in Chinese). Accessed 24 Jan 2016.

22. Frank LD, Engelke PO. Health and community design: the impact of the built environment on physical activity. Washington, DC: Island Press; 2003.

23. Veitch J, Salmon J, Ball K, Crawford D, Timperio A. Do features of public open spaces vary between urban and rural areas? Prev Med. 2013;56(2):107-11.

24. Westgarth C, Christley RM, Christian HE. How might we increase physical activity through dog walking?: a comprehensive review of dog walking correlates. Int J Behav Nutr Phys Act. 2014;11:83. 
25. Toohey AM, Rock MJ. Unleashing their potential: a critical realist scoping review of the influence of dogs on physical activity for dog-owners and non-owners. Int J Behav Nutr Phys Act. 2011;8:46.

26. Hallal PC, Gomez LF, Parra DC, Lobelo F, Mosquera J, Florindo AA, et al. Lessons learned after 10 years of IPAQ use in Brazil and Colombia. J Phys Act Health. 2010;7(Suppl 2):S259-64.

27. Schofield G, Mummery K, Steele R. Dog ownership and human healthrelated physical activity: an epidemiologic study. Health Promot J Aust. 2005;16:15-9.

28. Directorate General of Budget Accounting and Statistics, Taiwan. Report on the survey of family income and expendture. Taipei: Executive Yuan; 2015. (in Chinese)

Submit your next manuscript to BioMed Central and we will help you at every step:

- We accept pre-submission inquiries

- Our selector tool helps you to find the most relevant journal

- We provide round the clock customer support

- Convenient online submission

- Thorough peer review

- Inclusion in PubMed and all major indexing services

- Maximum visibility for your research

Submit your manuscript at www.biomedcentral.com/submit
Biomed Central 